\title{
Effect of Hydrophobic Groups on Adsorption of Amino Acids to Solid Surfaces in Water
}

\author{
Akinori Fukushima ( $\nabla$ akinori@u-fukui.ac.jp ) \\ Fukui Daigaku https://orcid.org/0000-0002-1860-3008 \\ Masaya Hirano \\ University of Fukui: Fukui Daigaku

\section{Ryuichi Sato} \\ University of Fukui: Fukui Daigaku
}

\section{Research Article}

Keywords: Peptide, Metal surface, Molecular dynamics, Free energy

Posted Date: February 21st, 2022

DOI: https://doi.org/10.21203/rs.3.rs-1321094/v1

License: (1) This work is licensed under a Creative Commons Attribution 4.0 International License.

Read Full License 


\title{
Effect of Hydrophobic Groups on Adsorption of Amino Acids to Solid Surfaces in Water
}

$\underline{\text { Akinori Fukushima }^{1 *}}$, Masaya Hirano ${ }^{2}$, and Ryuichi Sato ${ }^{2}$

${ }^{1}$ Faculty of Engineering, University of Fukui, 3-9-1, Bunkyo, Fukui, Fukui 910-8507, Japan

${ }^{2}$ Graduate School of Engineering, University of Fukui, 3-9-1, Bunkyo, Fukui, Fukui 910-8507, Japan

$+81-776-27-8526$

*akinori@u-fukui.ac.jp

\begin{abstract}
:
We calculated the free energies of adsorption between a solid wall and various arginine derivatives in water using molecular dynamics simulations. We varied the hydrophobic group size of the amino acids in two ways and the hydrophilicity of the solid wall in five ways. The free energy of adsorption decreased by increasing the hydrophilicity of the solid wall, regardless of the size of the hydrophobic group. Amino acids with small hydrophobic groups adsorbed on the solid wall with the strongest hydrophilicity, but the free energy of the amino acids with large hydrophobic groups was relatively small. The free energy of adsorption in benzene solvent increased by increasing hydrophilicity of the solid wall, yet analogously to results in water solvent was independent of the size of the hydrophobic group. We attribute these differences in free energy to the effect of solvent molecules localized on the solid wall. Thus, these results may modulate peptide adsorption on a solid surface by the size of the peptide's hydrophobic groups.
\end{abstract}

Keywords: Peptide, Metal surface, Molecular dynamics, Free energy 


\section{Introduction}

Aptamers are DNA, RNA, and peptides that have a specific affinity for particular substances[1, 2], such as small molecules[3, 4], ions[5, 6], proteins[7, 8], cells[9, 10], and organisms[11, 12]. Aptamers have many advantages over antibodies, which otherwise have similar properties to aptamers. For example, aptamers are less immunogenic, have better specificity, have higher affinity to their targets, and exhibit less non-specific cross-reactivity than antibodies[13-15]. Because one selects aptamers in vitro, they can be used against a broad range of targets, such as toxic and non-immunogenic substances[16]. Compared with antibodies, aptamers are stable to heat[17] and $\mathrm{pH}[18]$, and they are resistant to organic solvents[19]. Thus, one can select target molecules under non-physiological conditions. Moreover, unlike antibodies, aptamers can undergo several denaturation/refolding cycles without loss of activity[20]. One can mass-produce aptamers by chemical synthesis at low production costs[16]. Accordingly, researchers use aptamers in a broad range of fields, such as diagnostics and therapy[1, 21-25], drug delivery[26-28], and biosensors[29-32].

Sensitive detection of metals based on the affinity and specificity of aptamers is an active field of research in environmental monitoring and medical diagnosis[2]. In medicine, the concentrations of various metals in vivo are biomarkers, measurable indicators of biological conditions. For example, titanium and its alloys see use in the production of many medical implants, such as artificial bones and joints. Recent studies suggest that titanium-based implants exhibit wear and corrosion in physiological environments, releasing titanium particles into surrounding tissues and blood. Because titanium is highly insoluble and can lead to tissue injury, researchers need accurate and facile measurements of titanium concentration in blood. However, precise measurements require technical equipment, rendering routine monitoring difficult[33]. Using aptamers in blood tests would enable measuring the concentration of various metals, such as titanium, in blood at a low cost. This would solve the problems associated with conventional testing methods. Thus, it is necessary to develop aptamers with specific and high affinity for various metals.

Researchers generally develop novel peptide aptamers by phage display[34-36], which screens 
peptides that have a high affinity for targets from peptide libraries comprised of a large number of peptides that have different amino acid sequences. Researchers have developed numerous peptide aptamers that have a specific affinity for various inorganic surfaces[37-44]. Sano et al. identified a peptide TBP-1 (RKLPDAPGMHTW) that specifically binds to Ti by using phage display and suggested that RKLPDA (minTBP-1) in the peptide sequence is pertinent to binding to the Ti surface. Initial studies on the binding affinity suggest that minTBP-1 has affinities for $\mathrm{Ti}, \mathrm{Si}$, and $\mathrm{Ag}$; but not for $\mathrm{Au}, \mathrm{Cr}, \mathrm{Pt}, \mathrm{Sn}, \mathrm{Zn}, \mathrm{Cu}$, and $\mathrm{Fe}[45]$. Regarding the roles of amino acid residues in the minTBP-1 sequence, alanine substitution experiments suggest that mutation of a neutral proline in the sequence decreases the affinity.

In contrast, mutation of a charged lysine in the sequence increases the affinity[46]. Skelton et al. obtained similar results using molecular dynamics simulations. They suggested that changes in the flexibility of minTBP-1 (due to mutations of proline) and changes in the stability of the molecular structure in the adsorbed state (due to mutations of lysine) are pertinent factors. Furthermore, the interactions of the charged residues in the sequence-Arg, Asp, and Lys - with the water layers structured on the $\mathrm{TiO}_{2}$ surface drive the initial stage of adsorption of minTBP-1[47]. These results suggest the importance of interfacial water layers in interactions between peptides and $\mathrm{TiO}_{2}$ surfaces. Schneider et al. performed molecular dynamics simulations to analyze the adsorption of minTBP-1 on $\mathrm{TiO}_{2}$ and $\mathrm{SiO}_{2}$ surfaces[48]. They suggested the pertinence of hydrophilic and hydrophobic residues to optimize matching with the density oscillations of the interfacial water molecules. Sultan et al. suggested the pertinence of peptide structures and sequences on binding affinity and evaluated various titanium-binding peptides Ti-1 (QPYLFATDSLIK) and Ti-2 (GHTHYHAVRTQT), from the standpoint of entropy and enthalpy. These two peptide sequences differ substantially in the overall balance between hydrophobic and charged residues. Hydropathy scores indicate that Ti-1 shows stronger hydrophobicity. However, quartz crystal microbalance experiments show that these two peptides have similar binding affinities for the $\mathrm{TiO}_{2}$ surface. Molecular dynamics simulations suggest that Ti-1 is an entropically driven binder, with no strong anchor residues, whereas Ti-2 is an 
enthalpically guided binder, featuring a high number of periodically spaced anchor residues along the chain length[49].

Researchers have also studied the mechanisms of the interactions between single amino acids and inorganic surfaces. Sultan et al. performed molecular dynamics simulations to calculate the adsorption free energy of various amino acid side chain analogues on negatively charged $\mathrm{TiO}_{2}$ surfaces. They suggested that charged side chain analogues have strong affinities, whereas uncharged side chain analogues show weak or repulsive interactions[50]. Using molecular dynamics simulations, Erik et al. calculated the adsorption free energy of 19 amino acid side chains on a TiO2 surface. They suggested that polar amino acid side chains have affinities for the surface, whereas hydrophobic amino acid side chains have low affinities[51]. Experimental and simulation studies indicate that charged amino acids (Arg, Lys, Asp, and Gln) and, to a lesser extent, polar amino acids (Ser, Thr, Asn, Gln, and Tyr) have strong binding affinity for $\mathrm{TiO}_{2}$ surfaces, whereas hydrophobic (Val, Leu, Ile, and Phe) residues exhibit negligible binding affinity[49, 52-57].

Despite many studies on the interactions between biomolecules and inorganic surfaces, researchers still do not fully understand why particular peptides have specific binding affinity for inorganic surfaces. By elucidating the fundamentals of specific recognition between peptides and inorganic surfaces, one can rationally design novel peptide aptamers based on the properties of the target inorganic surfaces, which will reduce research and development costs. Therefore, it is necessary to understand how the properties of peptides, which are determined by their steric structures and amino acid sequences, affect their interactions with inorganic surfaces and how one can tailor these properties to modulate affinity.

We focused on hydrophobic groups in amino acids to understand their affinity for solid walls. We used virtual amino acid molecules based on arginine. In physiological environments, metal surfaces are oxidized and positively or negatively charged[58]. Assuming a negatively charged metal solid surface, we simulated virtual solid walls with downward dipole moments on the surface. We performed molecular dynamics simulations to evaluate changes in the adsorption states of amino 
acids and changes in the magnitude of the dipole moments by calculating the free energy.

\section{Computational Details}

Figure 1 shows the structures of the virtual amino acids based on arginine used in this study. The amino acid derivatives consist of a hydrophobic carbon chain with an amine that is bonded to the carboxy group of the main chain of arginine, thus constituting an amide bond. The number of carbon atoms in the hydrophobic chain is 1 and 16 . We named two carbon atoms in amino acids. Carbon atoms at the end of the hydrophobic carbon chain are called $\mathrm{C}_{1}$, and carbons bonded with three hydrogen atoms are called $\mathrm{C}_{2}$. Figure 2 shows the system used in the simulation and the definition of the reaction coordinate. Figure 2(a) shows the simulation system. The solid wall consists of one virtual atom based on FCC's crystal structure. The orientation of the surface where the virtual molecules adsorb is the (111) plane. We prepared a supercell volume, $39.0 \AA \times 29.4 \AA \times 300 \AA$. For solvent molecules, we used 5000 water molecules. The number of virtual molecules was one. We used periodic boundary conditions in all directions. Figure 2(b) shows the schematic diagram of the reaction path. We employed a difference of the z-coordinate between the surface of the solid wall and the centre of mass of the amino acid.

We determined the partial atomic charge of the amino acids by fitting the electron density calculated from optimized structures using the restrained electrostatic potential method. All electronic structure calculations were carried out based on the density functional theory using gaussian16[59]. We used B3LYP $[60]$ functionals as the exchange-correlation functionals. Moreover, the $6-31 \mathrm{G}^{* *}$ basis set was used for all atoms. We used harmonic oscillators for the bond, angle, and dihedral potentials. The $\mathrm{SPC} / \mathrm{E}[61]$ model was used for water molecules and General Amber Force Field[62] for the amino acid. We evaluated interaction parameters between various atoms using the Lorentz-Berthelot mixing

rule. We set the Coulomb and Lennard-Jones potential cutoffs at $10 \AA$ and the dipole potential cutoff at $15 \AA$. The particle-particle-particle-mesh methods[63] were used to calculate the distance beyond the cutoff distance of the Coulomb potential. We constrained the bond lengths and bond angles of the 
water molecules by the SHAKE[64] method.

To maintain the structure of the solid wall, we used a harmonic oscillator potential between virtual atoms in the unit cell (Figure 2):

$\phi_{H \text { armonic }}=K\left(r-r_{0}\right)^{2}$

where $r$ is the distance to atoms, $r_{0}$ is the equilibrium distance between two atoms (4.08 $\AA$ ), and $K$ is a constant $\left(500 \mathrm{kcal} / \mathrm{mol} / \AA^{2}\right)$. Assuming a negatively charged surface, all atoms in the most outside layers were assigned downward dipole moments in the simulation. We changed the magnitude of the dipole moment $(D)$ from $0.0 e \AA, 0.5 e \AA, 1.0 e \AA, 1.5 e \AA$, and $2.0 e \AA$. To represent the electric field on the solid wall by this dipole moment, the charges of all the atoms constituting the solid wall were assumed to be zero. We set the parameters $\varepsilon$ and $\sigma$ of the virtual atoms of the solid walls to appropriate values to prevent solvent molecules from entering the solid wall.

We carried out molecular dynamics simulations with LAMMPS[65]. In the initial state, virtual molecules were placed at $10 \AA$ from the solid wall surface to its centre of mass, and water molecules were randomly placed in the region of 25-100 $\AA$ from the solid wall surface. We set the time step for the numerical integration to $1 \mathrm{fs}$. We calculated the free energy using the umbrella sampling method [66] and the weighted histogram analysis method[67]. In this study, the reaction coordinates were defined as the distance from the solid wall surface $\left(z_{w}\right)$ to the centre of gravity of the amino acid $\left(z_{p}\right)$ as shown in figure 2(b). We varied the reaction coordinates from $4.8 \AA$ to $22.8 \AA$ in increments of 1.0 $\AA$ up to the bulk solvent. To control the reaction coordinates, a harmonic potential was imposed to constrain only the distance in the z-direction. Therefore, the amino acids are free to move in the $x y$ direction.

At the first step of the simulation, to create a stable state constrained by the harmonic potential, we set the spring constant for the harmonic potential to control the reaction coordinate to 1000 $\mathrm{kcal} / \mathrm{mol} / \AA^{2}$ and calculated $200 \mathrm{ps}$ in the $N V T$ ensemble at a temperature of $300 \mathrm{~K}$. Moreover, we add 
extra body force to all water molecules. Then, we removed the additional body force, changed the spring constant to $100 \mathrm{kcal} / \mathrm{mol} / \AA^{2}$, and ran the simulation for $200 \mathrm{ps}$. After obtaining the equilibrium state, we set the spring constant for the harmonic potential to control the reaction coordinate to 1 $\mathrm{kcal} / \mathrm{mol} / \AA^{2}$ and calculated 200ps for sampling.

\section{Results and Discussion}

At first, we discuss the adsorption free energy. The free energy at each reaction coordinate are shown in figure 3. Panel (a) shows the free energy of the amino acid with $n=1$, and panel (b) shows the free energy of the amino acid with $n=16$. The red line indicates results in the case of $D=0 e \AA$, the green line indicates results in the case of $D=0.5 \mathrm{e} \AA$, the blue line indicates results in the case of $D$ $=1.0 \mathrm{e} \AA$, the purple line indicates results in the case of $D=1.5 e \AA$, and the black line indicates results in the case of $D=2.0 e \AA$, respectively. The free energy at $z=22.8 \AA$ was set to 0 , where the amino acid was sufficiently far from the solid wall to ignore the interaction between the amino acid and the solid wall. The horizontal axis shows the reaction path of amino acid. For $n=1$, the free energy curve can be divided into two types. One type can be seen when the dipole moment is $0 e \AA, 0.5 e \AA$, and 1 $e \AA$. In this type, the stable point is located where the distance parameter is $5 \mathrm{~A}$. On the other hand, for dipole moments of $1.5 e \AA$ and $2.0 e \AA$, there is an adsorption point near $7 \AA$ (the local stable state in $2.0 e \AA)$. These different adsorption points indicate that the adsorption state may change with the magnitude of the dipole moment. Even when $\mathrm{n}$ is 16 , the free energy curve can be divided into two parts. One is the type with adsorption points in the region near $5 \AA$, as in the case of $n=1(D=0 e \AA$, $0.5 e \AA, 1.0 e \AA$ ), and the other is the type with adsorption points in the area farthest from the wall $(D=1.5 e \AA, 2.0 e \AA)$.

Figure 4 shows the magnitude of the free energy of the adsorbed state at each dipole for the amino acid with $n=1$ and $n=16$. When the dipole is $0 e \AA$, the adsorption energy increases in proportion to the number of carbons in the virtual molecule. However, as the dipole increases, the magnitude of the adsorption free energy of the virtual molecule with $n=16$ becomes smaller than that of the amino acids 
with $n=1$. This indicates that the decrease in the magnitude of the free energy is dependent on the size of the hydrophobic component of the amino acid. On the other hand, the rate of increase in adsorption free energy of $n=16$ amino acids with the sizeable hydrophobic component is more significant than that of $n=1$ amino acid. This indicates that the adsorption on the solid wall can be regulated by adjusting the size of the hydrophobic component.

Next, we discuss adsorption geometry. we focused on the difference of the stable structure between the magnitude of the dipole moment. Figure 5 shows the probability distribution of two carbon atoms. Panel (a) indicates results of $n=1$, and panel (b) indicates results of $n=16$. We defined the distance between carbon atom $\mathrm{C}_{1}$ at the bonded end of the side chain of arginine and the solid wall and the distance between the free terminus carbon atom $\mathrm{C}_{2}$ at the end of the hydrophobic component of the amino acid and the solid wall as parameters as shown in figure 1 . The horizontal axis means parameters defined above, and the vertical axis represents the probability distribution. Purple lines indicate results of $\mathrm{C}_{1}$, and red lines indicate results of $\mathrm{C}_{2}$, respectively. Moreover, solid lines indicate results of $D=0 e \AA$, and dashed lines indicate results of $D=2 e \AA$. In the case of $\mathrm{n}=1$, Comparing the case of $D=0 e \AA$ with the case of $D=2 e \AA$, we can see that the position of $\mathrm{C}_{2}$ does not change, but the position of $\mathrm{C}_{1}$ differs significantly. This indicates that when $D$ is equal to $0 e \AA$, the molecule is parallel to the solid wall, while when $D$ is equal to $2 e \AA$, the molecule is perpendicular to the solid wall. We also show the schematic diagram of the adsorption structure at $n=1$ in figure 6 . Panel (a) indicates that the molecule is parallel to the solid wall $(D=0 e \AA)$, and panel (b) indicates that the molecule is perpendicular to the solid wall $(D=2 e \AA)$. As shown in panel (a), when the dipole moment of the solid wall is zero, since the molecule is adsorbed on the solid wall via the non-coulombic interaction, atoms that compose the molecule are adsorbed in a state where the distance between them and the solid wall becomes small. On the other hand, in the case of the $D=2 e \AA$, the dipole interacts strongly with charged atoms, like oxygen in water and nitrogen in our molecule. As a result, carbon atoms, which have fewer charges, are inhibited by water to close the solid wall. This fact indicates that the adsorption of amino acids on solid walls in water is likely to be determined by the magnitude of the 
interaction between the solid surface and water molecules and charged sites such as nitrogen atoms, i.e., the size of the surface dipole, and the length of the carbon chain with which these interactions are small.

\section{Conclusions}

We focused on the effect of hydrophobic groups in amino acid molecules to obtain fundamental insight on modulating peptide binding affinity to solid walls. We modelled amino acids with differentsized hydrophobic groups based on arginine, a polar amino acid with a high affinity for inorganic surfaces. To investigate the effect of the difference of the surface dipole on the adsorption free energy, we simulated a solid wall with a downward dipole moment on the surface. We performed molecular dynamics simulations to evaluate the change in the adsorption state of amino acids and the free energy due to the difference in the dipole moment on the solid wall. As increasing the dipole moment of the solid surface, the free energy of the amino acid adsorption decreased regardless of the size of the hydrophobic group. Still, the increasing rate of the free energy of the $n=1$ amino acid was more significant than that of the $n=16$ amino acid. As a result, when the magnitude of the dipole moment is more important than $1 e \AA$, the free energy of the amino acid with a large hydrophobic group is smaller than that of the amino acid with a small hydrophobic group. This result suggests that the adsorption state may be controlled by the size of the hydrophobic group. Next, we focus on the adsorption geometry of the amino acid. In the case of $n=1$, when $D$ is smaller than $1 e \AA$, the amino acid is adsorbed parallel to the solid wall. On the other hand, when $D$ is higher than $1.5 e \AA$, the amino acid is adsorbed perpendicular to the solid wall. This difference is caused by the coulombic interaction between water molecules and the solid wall. This fact indicates that the adsorption of amino acids on solid walls in water is likely to be determined by the magnitude of the interaction between the solid surface and water molecules and charged sites such as nitrogen atoms, i.e., the size of the surface dipole, and the length of the carbon chain with which these interactions are small. 


\section{Acknowledgment}

In this research work, we used the supercomputer of ACCMS, Kyoto University.

\section{References}

1. Kadioglu O, Efferth T (2016) Peptide aptamer identified by molecular docking targeting translationally controlled tumor protein in leukemia cells. Invest New Drugs 34:515-521. https://doi.org/10.1007/s10637-016-0339-6

2. Farzin L, Shamsipur M, Sheibani S (2017) A review: Aptamer-based analytical strategies using the nanomaterials for environmental and human monitoring of toxic heavy metals. Talanta 174:619-627. https://doi.org/https://doi.org/10.1016/j.talanta.2017.06.066

3. Shamsipur M, Farzin L, Tabrizi MA, Shanehsaz M (2016) CdTe amplification nanoplatforms capped with thioglycolic acid for electrochemical aptasensing of ultra-traces of ATP. Mater Sci Eng C 69:1354-1360. https://doi.org/https://doi.org/10.1016/j.msec.2016.08.038

4. Zhang S, Wang L, Liu M, et al (2016) A novel, label-free fluorescent aptasensor for cocaine detection based on a G-quadruplex and ruthenium polypyridyl complex molecular light switch. Anal Methods 8:3740-3746. https://doi.org/10.1039/C6AY00231E

5. Zhou W, Ding J, Liu J (2017) 2-Aminopurine-modified DNA homopolymers for robust and sensitive detection of mercury and silver. Biosens Bioelectron 87:171-177. https://doi.org/https://doi.org/10.1016/j.bios.2016.08.033

6. Guo Y, Chen Y, Wei Y, et al (2015) Label-free fluorescent aptasensor for potassium ion using structure-switching aptamers and berberine. Spectrochim Acta Part A Mol Biomol Spectrosc 136:1635-1641. https://doi.org/https://doi.org/10.1016/j.saa.2014.10.058

7. Amouzadeh Tabrizi M, Shamsipur M, Farzin L (2015) A high sensitive electrochemical aptasensor for the determination of VEGF165 in serum of lung cancer patient. Biosens Bioelectron 74:764-769. https://doi.org/https://doi.org/10.1016/j.bios.2015.07.032

8. Shamsipur M, Farzin L, Tabrizi MA (2016) Ultrasensitive aptamer-based on-off assay for 
lysozyme using a glassy carbon electrode modified with gold nanoparticles and electrochemically reduced graphene oxide. Microchim Acta 183:2733-2743. https://doi.org/10.1007/s00604-016-1920-6

9. Mir TA, Yoon J-H, Gurudatt NG, et al (2015) Ultrasensitive cytosensing based on an aptamer modified nanobiosensor with a bioconjugate: Detection of human non-small-cell lung cancer cells. Biosens Bioelectron 74:594-600.

https://doi.org/https://doi.org/10.1016/j.bios.2015.07.012

10. Wang T, Liu J, Gu X, et al (2015) Label-free electrochemical aptasensor constructed by layer-by-layer technology for sensitive and selective detection of cancer cells. Anal Chim Acta 882:32-37. https://doi.org/https://doi.org/10.1016/j.aca.2015.05.008

11. Labib M, Zamay AS, Kolovskaya OS, et al (2012) Aptamer-Based Viability Impedimetric Sensor for Bacteria. Anal Chem 84:8966-8969. https://doi.org/10.1021/ac302902s

12. Labib M, Zamay AS, Muharemagic D, et al (2012) Aptamer-Based Viability Impedimetric Sensor for Viruses. Anal Chem 84:1813-1816. https://doi.org/10.1021/ac203412m

13. Collett JR, Cho EJ, Ellington AD (2005) Production and processing of aptamer microarrays. Methods 37:4-15. https://doi.org/https://doi.org/10.1016/j.ymeth.2005.05.009

14. Mayer G (2009) The Chemical Biology of Aptamers. Angew Chemie Int Ed 48:2672-2689. https://doi.org/https://doi.org/10.1002/anie.200804643

15. Templin MF, Stoll D, Schrenk M, et al (2002) Protein microarray technology. Trends Biotechnol 20:160-166. https://doi.org/https://doi.org/10.1016/S0167-7799(01)01910-2

16. Kadioglu O, Malczyk AH, Greten HJ, Efferth T (2015) Aptamers as a novel tool for diagnostics and therapy. Invest New Drugs 33:513-520. https://doi.org/10.1007/s10637-0150213-y

17. Langer R (1998) Drug delivery and targeting. Nature 392:5-10

18. Farokhzad OC, Cheng J, Teply BA, et al (2006) Targeted nanoparticle-aptamer bioconjugates for cancer chemotherapy in vivo. Proc Natl Acad Sci 103:6315 LP - 6320. 
https://doi.org/10.1073/pnas.0601755103

19. Wilson C, Szostak JW (1998) Isolation of a fluorophore-specific DNA aptamer with weak redox activity. Chem Biol 5:609-617. https://doi.org/https://doi.org/10.1016/S1074$5521(98) 90289-7$

20. Liss M, Petersen B, Wolf H, Prohaska E (2002) An Aptamer-Based Quartz Crystal Protein Biosensor. Anal Chem 74:4488-4495. https://doi.org/10.1021/ac011294p

21. Han J, Gao L, Wang J, Wang J (2020) Application and development of aptamer in cancer: from clinical diagnosis to cancer therapy. J Cancer 11:6902-6915. https://doi.org/10.7150/jca.49532

22. Nimjee SM, Rusconi CP, Sullenger BA (2004) Aptamers: An Emerging Class of Therapeutics. Annu Rev Med 56:555-583. https://doi.org/10.1146/annurev.med.56.062904.144915

23. Xing H, Hwang K, Li J, et al (2014) DNA aptamer technology for personalized medicine. Curr Opin Chem Eng 4:79-87. https://doi.org/https://doi.org/10.1016/j.coche.2014.01.007

24. Kanwar JR, Shankaranarayanan JS, Gurudevan S, Kanwar RK (2014) Aptamer-based therapeutics of the past, present and future: from the perspective of eye-related diseases. Drug Discov Today 19:1309-1321. https://doi.org/https://doi.org/10.1016/j.drudis.2014.02.009

25. Santosh B, Yadava PK (2014) Nucleic acid aptamers: research tools in disease diagnostics and therapeutics. Biomed Res Int 2014:540451. https://doi.org/10.1155/2014/540451

26. Wang C, Liu B, Lu J, et al (2014) Strategies for combination of aptamer and targeted drug delivery. J Nanosci Nanotechnol 14:501-512. https://doi.org/10.1166/jnn.2014.8746

27. Zhou J, Rossi JJ (2014) Cell-type-specific, Aptamer-functionalized Agents for Targeted Disease Therapy. Mol Ther - Nucleic Acids 3:e169. https://doi.org/https://doi.org/10.1038/mtna.2014.21

28. Xiang D, Shigdar S, Qiao G, et al (2015) Nucleic acid aptamer-guided cancer therapeutics 
and diagnostics: the next generation of cancer medicine. Theranostics 5:23-42. https://doi.org/10.7150/thno.10202

29. Kim YS, Gu MB (2014) Advances in aptamer screening and small molecule aptasensors. Adv Biochem Eng Biotechnol 140:29-67. https://doi.org/10.1007/10_2013_225

30. MacKay S, Wishart D, Xing JZ, Chen J (2014) Developing Trends in Aptamer-Based Biosensor Devices and Their Applications. IEEE Trans Biomed Circuits Syst 8:4-14. https://doi.org/10.1109/TBCAS.2014.2304718

31. Zhou W, Huang P-JJ, Ding J, Liu J (2014) Aptamer-based biosensors for biomedical diagnostics. Analyst 139:2627-2640. https://doi.org/10.1039/c4an00132j

32. Reverdatto S, Burz DS, Shekhtman A (2015) Peptide aptamers: development and applications. Curr Top Med Chem 15:1082-1101. https://doi.org/10.2174/1568026615666150413153143

33. Swiatkowska I, Martin N, Hart AJ (2019) Blood titanium level as a biomarker of orthopaedic implant wear. J trace Elem Med Biol organ Soc $\quad$ Miner Trace Elem 53:120-128. https://doi.org/10.1016/j.jtemb.2019.02.013

34. Smith GP, Petrenko VA (1997) Phage Display. Chem Rev 97:391-410. https://doi.org/10.1021/cr960065d

35. Smith GP (1985) Filamentous fusion phage: novel expression vectors that display cloned antigens on the virion surface. Science 228:1315-1317. https://doi.org/10.1126/science.4001944

36. Scott JK, Smith GP (1990) Searching for peptide ligands with an epitope library. Science (80- ) 249:386 LP - 390. https://doi.org/10.1126/science.1696028

37. Brown S (1992) Engineered iron oxide-adhesion mutants of the Escherichia coli phage lambda receptor. Proc Natl Acad Sci U S A 89:8651-8655. https://doi.org/10.1073/pnas.89.18.8651

38. Brown S (1997) Metal-recognition by repeating polypeptides. Nat Biotechnol 15:269-272. 
https://doi.org/10.1038/nbt0397-269

39. Whaley SR, English DS, Hu EL, et al (2000) Selection of peptides with semiconductor binding specificity for directed nanocrystal assembly. Nature 405:665-668. https://doi.org/10.1038/35015043

40. Naik RR, Stringer SJ, Agarwal G, et al (2002) Biomimetic synthesis and patterning of silver nanoparticles. Nat Mater 1:169-172. https://doi.org/10.1038/nmat758

41. Sarikaya M, Tamerler C, Jen AK-Y, et al (2003) Molecular biomimetics: nanotechnology through biology. Nat Mater 2:577-585. https://doi.org/10.1038/nmat964

42. Kjærgaard K, Sørensen JK, Schembri MA, Klemm P (2000) Sequestration of Zinc Oxide by Fimbrial Designer Chelators. Appl Environ Microbiol 66:10 LP - 14. https://doi.org/10.1128/AEM.66.1.10-14.2000

43. Naik RR, Brott LL, Clarson SJ, Stone MO (2002) Silica-precipitating peptides isolated from a combinatorial phage display peptide library. J Nanosci Nanotechnol 2:95-100. https://doi.org/10.1166/jnn.2002.074

44. Lee S-W, Mao C, Flynn CE, Belcher AM (2002) Ordering of quantum dots using genetically engineered viruses. Science 296:892-895. https://doi.org/10.1126/science.1068054

45. Sano K-I, Sasaki H, Shiba K (2005) Specificity and Biomineralization Activities of TiBinding Peptide-1 (TBP-1). Langmuir 21:3090-3095. https://doi.org/10.1021/la047428m

46. Sano K-I, Shiba K (2003) A Hexapeptide Motif that Electrostatically Binds to the Surface of Titanium. J Am Chem Soc 125:14234-14235. https://doi.org/10.1021/ja038414q

47. Skelton AA, Liang T, Walsh TR (2009) Interplay of Sequence, Conformation, and Binding at the Peptide-Titania Interface as Mediated by Water. ACS Appl Mater Interfaces 1:14821491. https://doi.org/10.1021/am9001666

48. Schneider J, Ciacchi LC (2012) Specific material recognition by small peptides mediated by the interfacial solvent structure. J Am Chem Soc 134:2407-2413. https://doi.org/10.1021/JA210744G 
49. Sultan AM, Westcott ZC, Hughes ZE, et al (2016) Aqueous Peptide-TiO2 Interfaces: Isoenergetic Binding via Either Entropically or Enthalpically Driven Mechanisms. ACS Appl Mater Interfaces 8:18620-18630. https://doi.org/10.1021/acsami.6b05200

50. Sultan AM, Hughes ZE, Walsh TR (2014) Binding Affinities of Amino Acid Analogues at the Charged Aqueous Titania Interface: Implications for Titania-Binding Peptides. Langmuir 30:13321-13329. https://doi.org/10.1021/la503312d

51. Brandt EG, Lyubartsev AP (2015) Molecular Dynamics Simulations of Adsorption of Amino Acid Side Chain Analogues and a Titanium Binding Peptide on the TiO $<$ inf $>2<$ inf $>(100)$ Surface. J Phys Chem C 119:18126-18139. https://doi.org/10.1021/acs.jpcc.5b02670

52. Limo MJ, Sola-Rabada A, Boix E, et al (2018) Interactions between Metal Oxides and Biomolecules: from Fundamental Understanding to Applications. Chem Rev 118:1111811193. https://doi.org/10.1021/acs.chemrev.7b00660

53. Leader A, Mandler D, Reches M (2018) The role of hydrophobic, aromatic and electrostatic interactions between amino acid residues and a titanium dioxide surface. Phys Chem Chem Phys 20:29811-29816. https://doi.org/10.1039/C8CP05775C

54. Sampath J, Kullman A, Gebhart R, et al (2020) Molecular recognition and specificity of biomolecules to titanium dioxide from molecular dynamics simulations. npj Comput Mater 6:34. https://doi.org/10.1038/s41524-020-0288-7

55. Liu S, Meng X-Y, Perez-Aguilar JM, Zhou R (2016) An In Silico study of TiO2 nanoparticles interaction with twenty standard amino acids in aqueous solution. Sci Rep 6:37761. https://doi.org/10.1038/srep37761

56. YazdanYar A, Aschauer U, Bowen P (2018) Adsorption Free Energy of Single Amino Acids at the Rutile (110)/Water Interface Studied by Well-Tempered Metadynamics. J Phys Chem C 122:11355-11363. https://doi.org/10.1021/acs.jpcc.7b12614

57. Monti S, Walsh TR (2010) Free Energy Calculations of the Adsorption of Amino Acid Analogues at the Aqueous Titania Interface. J Phys Chem C 114:22197-22206. 
https://doi.org/10.1021/jp107859q

58. Kosmulski M (2016) Isoelectric points and points of zero charge of metal (hydr)oxides: 50years after Parks' review. Adv Colloid Interface Sci 238:1-61.

https://doi.org/https://doi.org/10.1016/j.cis.2016.10.005

59. Frisch MJ et al. (2016) Gaussian 09

60. Becke AD (1993) Density-functional thermochemistry. III. The role of exact exchange. J Chem Phys 98:5648-5652. https://doi.org/10.1063/1.464913

61. Berendsen HJC, Grigera JR, Straatsma TP (1987) The missing term in effective pair potentials. J Phys Chem 91:6269-6271

62. Wang J, Wolf RM, Caldwell JW, et al (2004) Development and testing of a general amber force field. J Comput Chem 25:1157-1174. https://doi.org/https://doi.org/10.1002/jcc.20035

63. Hockney RW, Eastwood JW (1966) Computer simulation using particles

64. Ryckaert JP, Ciccotti G, Berendsen HJC (1977) Numerical Integration of the Cartesian Equations of Motion of a System with Constraints: Molecular Dynamics of n-Alkanes. J Comput Phys 23:327-341. https://doi.org/10.1016/0021-9991(77)90098-5

65. Plimpton S (1995) Fast parallel algorithms for short-range molecular dynamics. J Comput Phys 117:1-19

66. Frenkel D, Smit B (2002) Understanding Molecular Simulation, 2nd ed. Academic Press, San Diego

67. Grossfield A "WHAM: the weighted histogram analysis method", version 2.0.11. http://membrane.urmc.rochester.edu/wordpress/?page_id=126 


\section{Ethics declarations}

Funding

This work was not supported by any funding.

Competing Interests

The authors declare that no competing interests.

Data Availability

All data will be available on reasonable request.

Code availability

Not applicable.

\section{Author Contributions}

Akinori Fukushima and Masaya Hirano performed the simulation and the data analysis and wrote a part of this article. Ryuichi Sato performed the simulation and the data analysis. All authors contributed to the general discussion. 


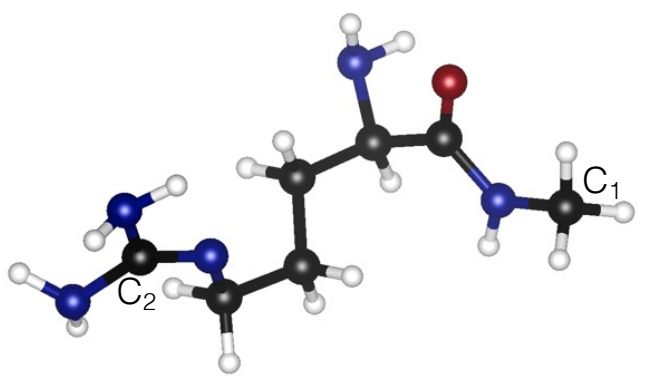

(a)

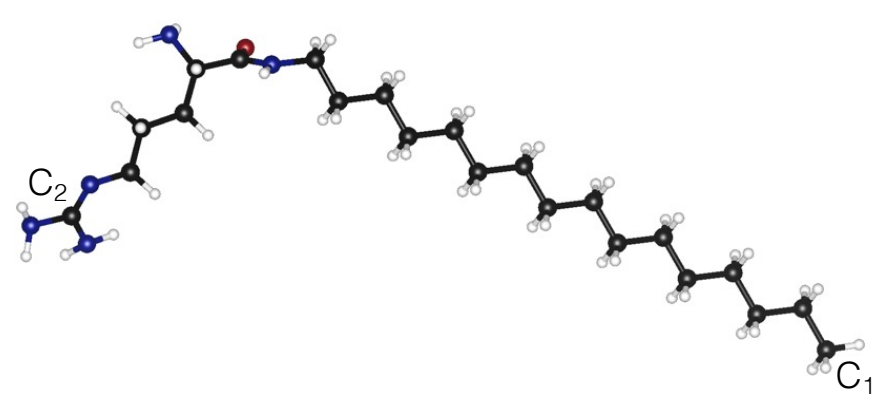

(b)

Figure 1 


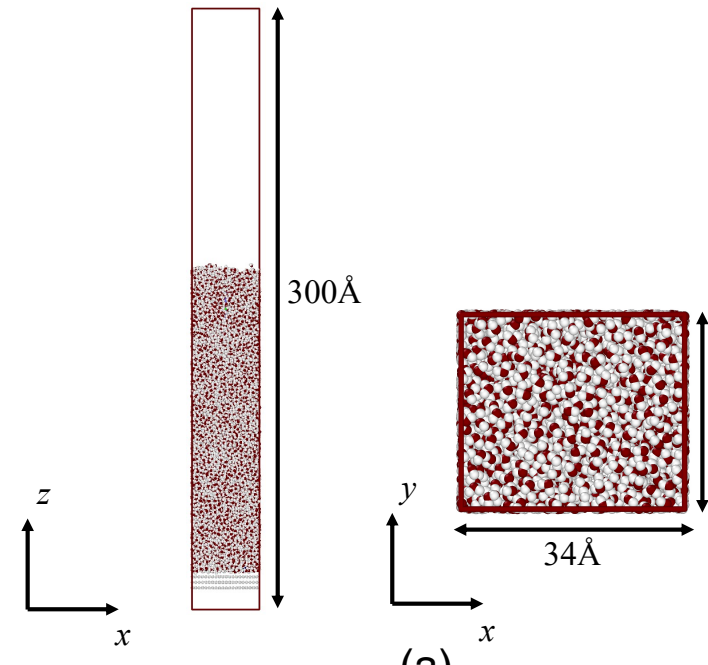

(a)

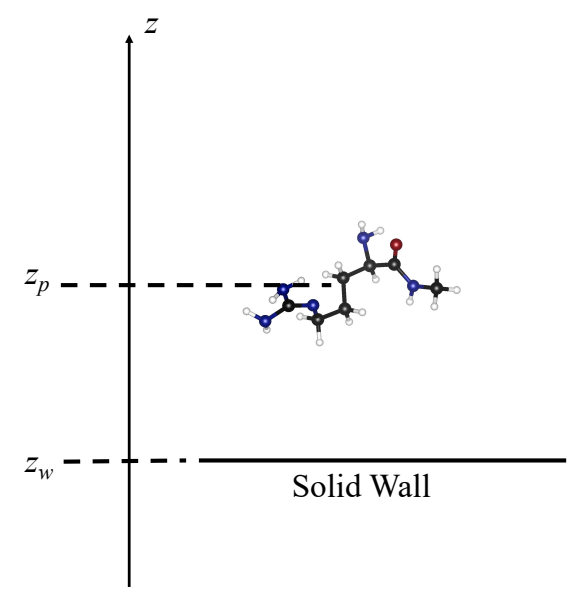

(b)

Figure 2 


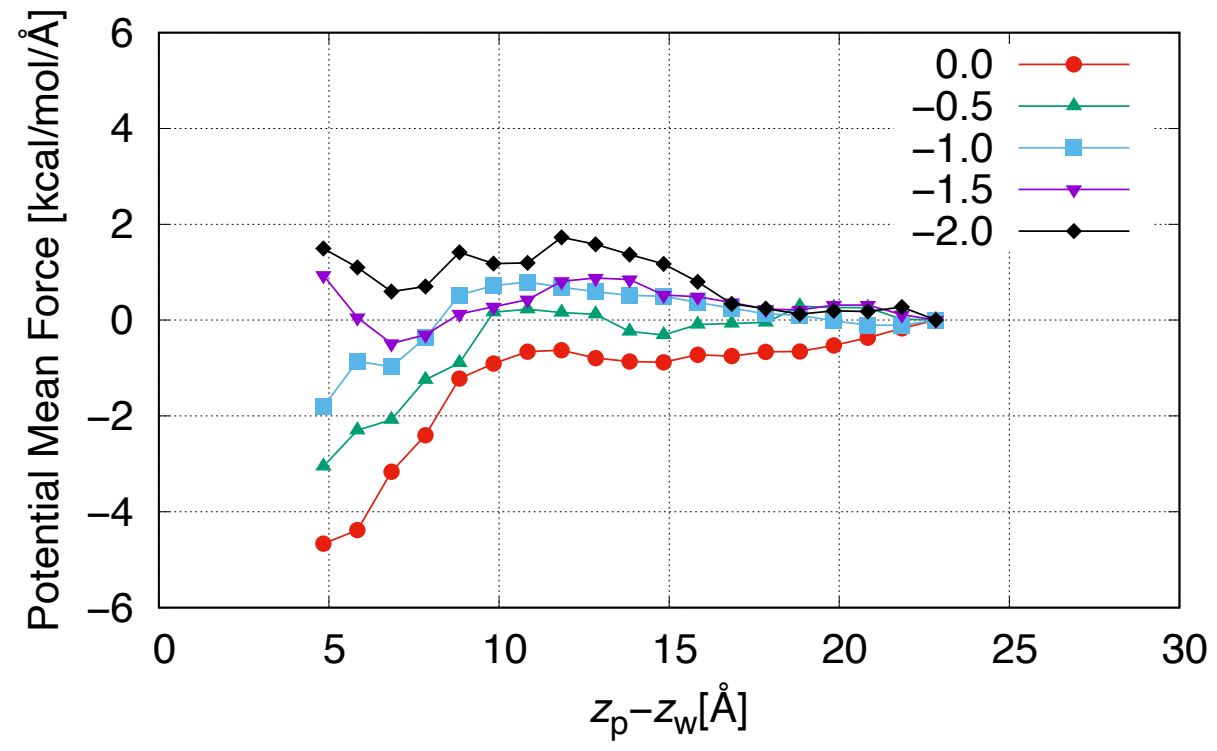

(a)

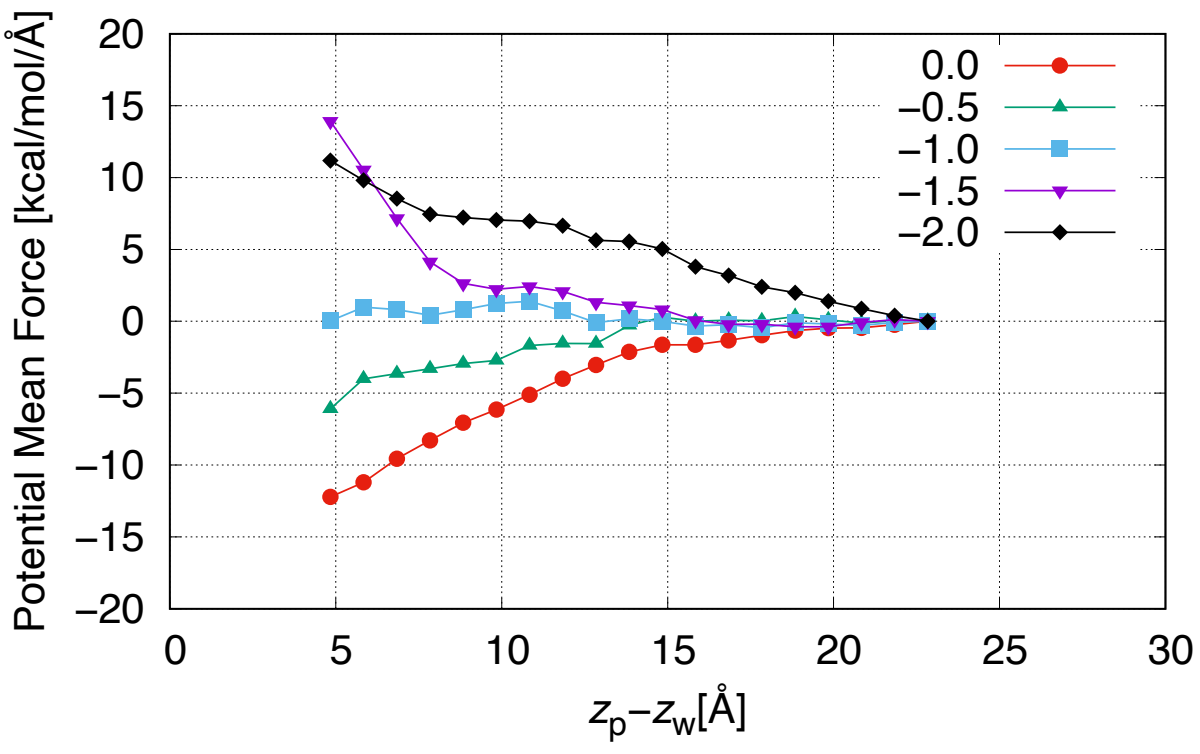

(b)

Figure 3 


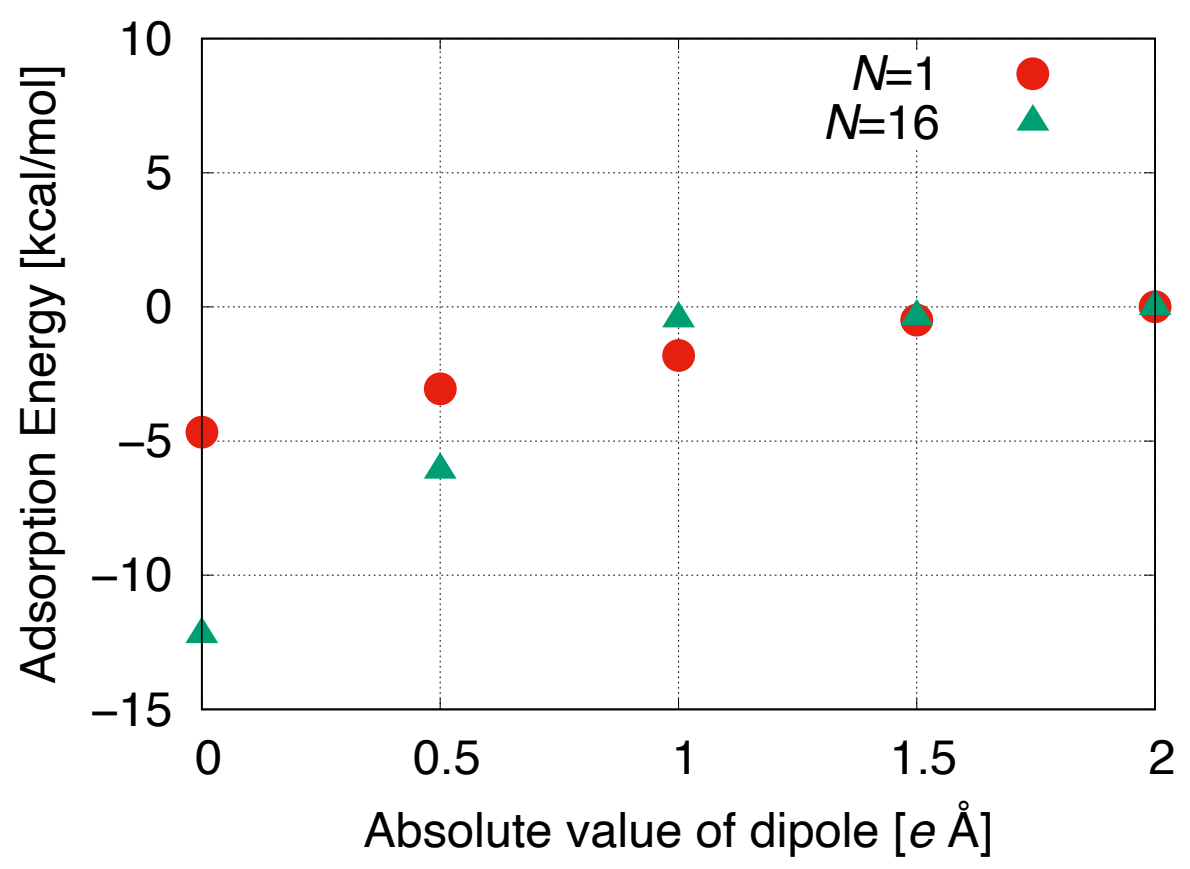

Figure 4 


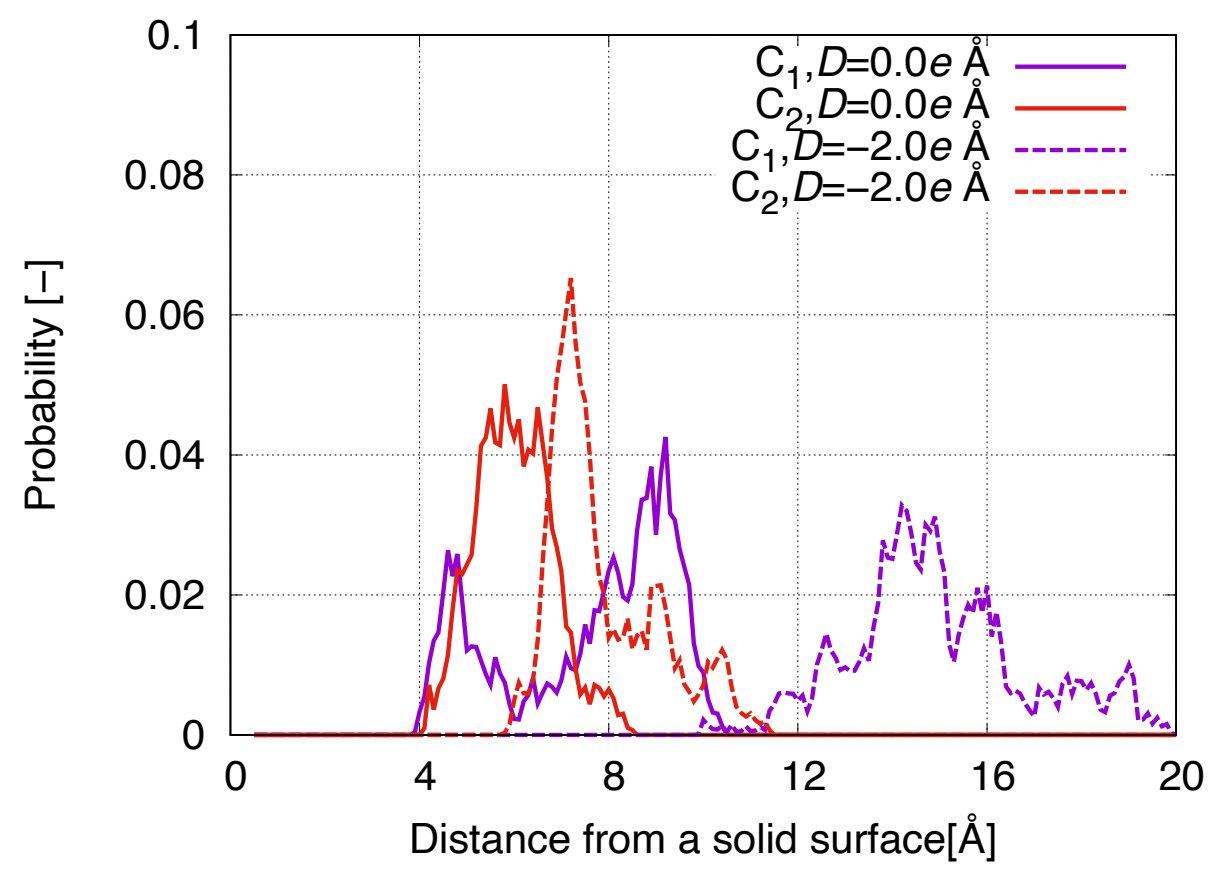

Figure 5 


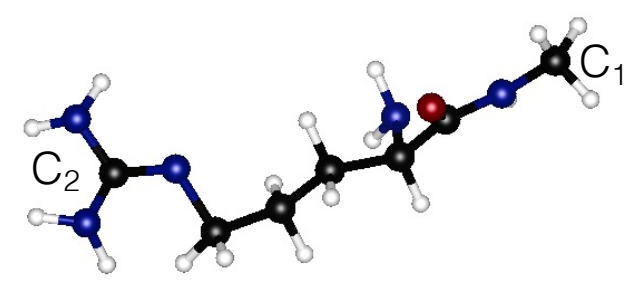

$\odot \odot \odot \odot$

(a)

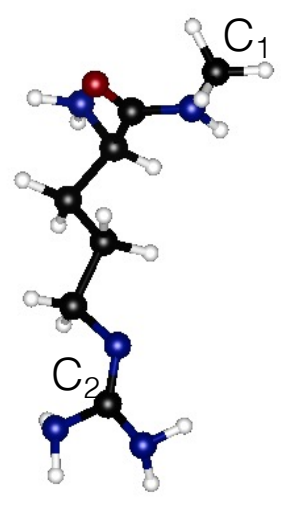

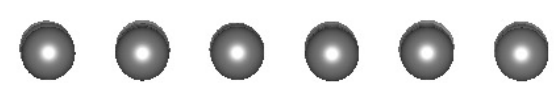

(b)

Figure 6 


\section{Figure captions}

Fig. 1 Chemical structures of molecules used in this study: (a) $n=1$; (b) $n=16$.

Fig. 2 Simulation systems and wall structures used in this study; (a) Simulation system. (b) Reaction pathway.

Fig. 3 Free energy diagram along the reaction path : (a) $n=1$; (b) $n=16$.

Fig. 4 Dependence of the adsorption free energy on the magnitude of the dipole moment. Red: $n=1$; purple: $n=16$.

Fig. 5 Probability density distributions: (a) $n=1$; (b) $n=16$.

Fig. 6 Schematic illustration of the adsorbed amino acid on the solid wall: (a) stable structure in $n=1$, $D=0 e \AA ;$ (b) local minimum structure in $n=1, D=2 e \AA$. 